\title{
Chemistry of aged garlic: Diversity of constituents in aged garlic extract and their production mechanisms via the combination of chemical and enzymatic reactions (Review)
}

\author{
YUKIHIRO KODERA, MASAHIRO KURITA, MASATO NAKAMOTO and TOSHIAKI MATSUTOMO
}

Central Research Institute, Wakunaga Pharmaceutical Co., Ltd., Akitakata-shi, Hiroshima 739-1195, Japan

Received August 27, 2019; Accepted September 30, 2019

DOI: $10.3892 / \mathrm{etm} .2019 .8393$

\begin{abstract}
Raw garlic contains characteristic compounds, such as $S$-alk(en)ylcysteine sulfoxides, $\gamma$-glutamyl- $S$-alk(en)ylcysteines and polysaccharides. These compounds undergo various transformation processes during the aging process. Among these compounds, the change of sulfur-containing molecules is diverse and time-dependent. Previously, by means of the liquid chromatography (LC)/LC-mass spectrometry (MS) method, a number of unidentified peaks corresponding to candidates of sulfur-containing molecules were detected in the chromatogram of aged garlic extract (AGE), and identified using MS and nuclear magnetic resonance (NMR). The production mechanisms of these compounds were then examined by model reactions and laboratory experiments mimicking the aging process. Three $\gamma$-glutamyl tripeptides [ $\gamma$-glutamyl- $\gamma$-glutamyl-S-methylcysteine, $\gamma$-glutamyl- $\gamma$-glutamyl-S-allylcysteine (GGSAC), $\gamma$-glutamyl- $\gamma$-glutamyl- $S$-1propenylcysteine], $\gamma$-glutamyl-S-allylmercaptocysteine (GSAMC) and cis-S-1-propenylcysteine (cis-S1PC) were isolated and identified. GGSAC was produced from GSAC through the enzymatic reaction catalyzed by $\gamma$-glutamyltranspeptidase (GGT), and two other tripeptides could be produced in similar reactions. GSAMC was produced by the reaction between $\gamma$-glutamyl dipeptides and allicin. Furthermore, GSAMC was a precursor compound of $S$-allylmercaptocysteine (SAMC), and thus it was produced from GSAMC by GGT. cis-S1PC was produced from trans-S1PC by the isomerization reaction. A number of other compounds were also identified, including Maillard reaction products; however, their production mechanisms have not been elucidated. In this review, we present the changes in characteristic constituents in raw garlic and garlic extract during the aging
\end{abstract}

Correspondence to: Dr Toshiaki Matsutomo, Central Research Institute, Wakunaga Pharmaceutical Co., Ltd., 1624 Shimokotachi, Koda-cho, Akitakata-shi, Hiroshima 739-1195, Japan

E-mail: matsutomo_t@wakunaga.co.jp

Key words: aged garlic extract, aging process, enzymatic reaction, sulfur-containing compounds, Maillard reaction products, $\gamma$-glutamyl transpeptidase process and discuss their production mechanisms involving the various chemical and enzymatic reactions.

\section{Contents}

1. Introduction

2. S-Alk(en)ylcysteines

3. cis-S-1-Propenylcysteine

4. $\gamma$-Glutamyl- $\gamma$-glutamyl-S-alk(en)ylcysteines

5. S-Allylmercaptocysteine

6. Hydrophobic sulfur compounds

7. Maillard reaction products

8. Sugars

9. Saponins

10. Compounds of which the production mechanisms have not been elucidated

11. Conclusions

\section{Introduction}

Allium plants incorporate the ultimate inorganic source, sulfate $\left(\mathrm{SO}^{2-}\right)$, fixed as L-cysteine molecule and produce several other organic sulfur compounds via various biosynthetic pathways, including glutamylation and glycylation to yield glutathione (GSH), deglycylation to yield $\gamma$-glutamyl-S-alk(en)ylcysteines, and $S$-oxygenation and deglutamylation to yield $S$-alk(en)ylcysteine sufloxides (Fig. 1A) (1-3). The biosynthesis and accumulation of these sulfur-containing compounds become the most active in one to two months prior to the harvest (4). Other constituents, such as common amino acids, carbohydrates, proteins and lipids, are also actively produced and accumulate during the same period when garlic bulbs grow rapidly (4). The resulting mature garlic is harvested from early summer through mid-summer to be used for various purposes, such as in foods, seasonings, medicinal treatments and others (4).

In the manufacturing of garlic products, raw garlic is processed through various methods, such as crushing, slicing, freeze-drying, heating, soaking, macerating, steam distillation, etc. Some constituents in raw garlic change to other compounds by processing, and the physical and chemical properties of preparation depend on the processing methods (5-7). Garlic oil, a yellow-brown-colored liquid with a sharp characteristic 
smell, is produced by the steam distillation method and its constituents are mainly composed of diallylpolysulfides (DASn) (Fig. 1B) (5,7). Oil macerate, a liquid form with a characteristic smell, is produced by the soaking of garlic in vegetable oil, and its constituents include unique sulfur compounds, ajoenes and vinyldithiins (Fig. 1B) $(5,7)$. Black garlic, mainly being supplied as a whole garlic bulb with an outer skin, is produced by the heating of garlic at $70-80^{\circ} \mathrm{C}$ for several months under high humidity conditions, and its constituents are claimed to contain Maillard reaction products and polyphenolic compounds $(8,9)$.

Another unique garlic preparation, aged garlic extract (AGE), is produced by the soaking of garlic in a water/ethanol mixture, and is thus subjected to natural extraction/aging for $>10$ months at room temperature. Its constituents include both hydrophilic and hydrophobic compounds, such as $S$-allylcysteine (SAC), $S$-1-propenylcysteine (S1PC), $S$-allylmercaptocysteine (SAMC), $N \alpha$-(1-deoxy-D-fructos-1 -yl)-L-arginine (Fru-Arg), DASn and phenolic compounds (Fig. 1C and E) (10-12). The biological properties of garlic have been evaluated by several researchers and garlic has been shown to have useful biological activities for the promotion of human health $(6,10,11,13)$. In addition, some characteristic constituents found in raw garlic, such as alliin, $\gamma$-glutamyl- $S$-allylcysteine (GSAC) and $\gamma$-glutamyl- $S$-1-propenylcysteine (GS1PC), are also found in AGE $(6,13)$. These diverse constituents found in AGE are thought to be produced by the processing conditions involving both physical and chemical factors, such as room temperature, a near neutral $\mathrm{pH}$, a suitable balance of hydrophilicity and hydrophobicity for promoting chemical/enzymatic reactions, suitable/sufficient processing time, etc. The compounds present in AGE can be produced via various and complex reactions. In particular, the complex chemistry of sulfur-containing compounds is considered to contribute mostly to the diversity of the constituents found in AGE. In order to reveal the production mechanisms of characteristic compounds found in AGE during the aging process, the model reaction and the laboratory experiments mimicking the aging process were conducted under the hypothesis of production mechanisms of identified compounds leading from the analytical results of key compounds fluctuations.

In this review, we present the changes of some characteristic compounds found in AGE during the aging process, and discuss their production mechanisms involving various chemical and enzymatic reactions. We also discuss associations between products and their precursor materials.

\section{S-Alk(en)ylcysteines}

Garlic accumulates $S$-alk(en)ylcysteine sulfoxides (SACOs; methiin, alliin and isoalliin) and $\gamma$-glutamyl- $S$-alk(en)ylcysteines [GSAkCs; $\gamma$-glutamyl- $S$-methylcysteine (GSMC), GSAC and GS1PC] as its characteristic sulfur constituents. GSAkCs abundantly exist in garlic cloves during the dormant period, from the time after harvest in summer to before sprouting in autumn (Figs. 1A and 2A) (1,4). However, the contents of these constituents decease when garlic cloves sense a decrease in environmental temperature, which acts as a stimulation signal to break the dormancy period, and they are gradually transformed into SACOs $(13,14)$. Furthermore, deoxy forms of SACOs
[S-methylcysteine (SMC), SAC and trans-S1PC] are hardly detected in raw garlic. Although only a trace amount of SAC is detected in raw garlic, the content of this compound increases during the processing of garlic $(13,14)$. The same phenomenon is observed for the generation of SMC and trans-S1PC. These compounds are produced from GSAkCs through the hydrolysis reaction by endogenous $\gamma$-glutamyltranspeptidase (GGT) in garlic that undergoes changes when chopped/sliced garlic is soaked into aqueous alcohol for $>10$ months, i.e., during the aging process (Figs. 1A, and $2 \mathrm{~A}$ and $\mathrm{C}$ ). GGT activity in AGE is detectable even after 300 days, although its activity is weak (10). Sun et al reported that GGT activity derived from garlic was significantly enhanced by glucose (Glc), aspartic acid (Asp) and cysteine (Cys) (15). Saccharides in raw garlic are mainly fructans that are composed of 1-2 glycosyl linkage polymer of fructose (Fru) with the reduction end of Glc (Fig. 1A). Raw garlic contains almost no free Glc; however, its content in AGE gradually increases with the progress of aging by the hydrolysis of fructan, including the release of Glc in the reduction end of fructan. The Asp content in raw garlic has been shown to be 0.2-6.1 mg/g-dry-weight (13). These constituents can promote the activity of GGT during the aging process.

In previous studies, when SMC, SAC and trans-S1PC were orally administered to experimental animals, they were rapidly absorbed, and exhibited excellent bioavailability, remaining stable in body fluids $(16,17)$. The beneficial effects of these compounds have been documented by a number of researchers $(13,18-24)$. The bioavailability of the oxide form of SAC, alliin, has been shown to be $<17 \%$ in a pharmacokinetic experiment using the radioisotope compound (25). Allicin, a transformation product of alliin, subsequently generates various compounds, such as DASn, ajoenes, vinyldithiins, etc. They have a low absorption rate and remain unstable in the body (25-28). The overall yields of these hydrophobic compounds are small as they transform into various compounds and their source compound in raw garlic is only alliin, i.e., two molecules of alliin transform into one molecule of allicin, diallyldisulfide (DADS) and dithiin, respectively, two molecules of ajoene are produced from three molecules of alliin, and these compounds produced subsequently transform into other compounds (13). On the other hand, one molecule of SAC is produced from one molecule of GSAC, and SMC and S1PC are produced in the same manner (13). Although produced $S$-alk(en)ylcysteines are generally stable in AGE, some portion of them are used to produce the other compounds, such as cis-S1PC, SAMC and Amadori compounds as described in the sections below.

The endogenous enzyme GGT which produces $S$-alk(en)ylcysteines is considered to be one of key factors for the diversity of constituents in AGE. The generation of water-soluble cysteine derivatives during the aging process may contribute to the promotion of human health.

\section{3. cis-S-1-Propenylcysteine}

The chemical and biological properties of S1PC have not been evaluated for a long period of time, although S1PC was found to be present in garlic preparations in the early 1960s (29). Recently, the fluctuation of its cis-and trans-form in AGE has been reported (30). S1PC is an isomer of SAC that is 
A<smiles>CS(=O)CC(N)C(=O)O</smiles><smiles>C=CCS(=O)CC(N)C(=O)O</smiles><smiles>CSCC(NC(=O)CCC(N)C(=O)O)C(=O)O</smiles>

Alliin r-Glutamyl-S-methylcysteine<smiles>C=CCSCC(NC(=O)CCC(N)C(=O)O)C(=O)O</smiles>
r-Glutamyl-S-allylcysteine (GSAC)<smiles>CC=CSCC(NC(=O)CCC(N)C(=O)O)C(=O)O</smiles>

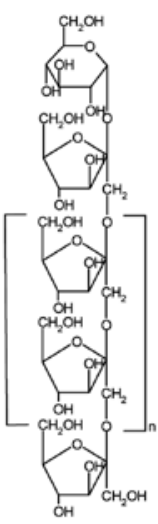

Fructan

B
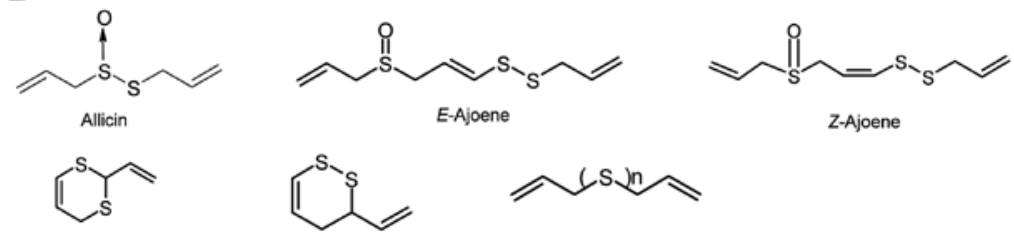

1,3-Vinyldithiin<smiles>C1=CSSC=CCC1</smiles><smiles>C=CCCCCC=C</smiles>

1,2-Vinyldithiin

Diallyipolysulfides (DASn)

C

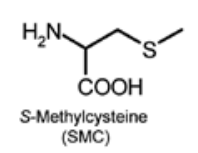<smiles>C=CCSCC(N)C(=O)O</smiles><smiles>C=CCSSCC(N)C(=O)O</smiles><smiles>C=CCSSSCC(N)C(=O)O</smiles><smiles>O=C(O)C1CCCCC1</smiles>
(S1PC)

(SAMC)<smiles>CSCC(NC(=O)CCC(NC(=O)CCC(N)=O)C(=O)O)C(=O)O</smiles>

$\mathrm{r}$-Glutamyl-v-glutamyl-S-methylcysteine
(GGSMC)

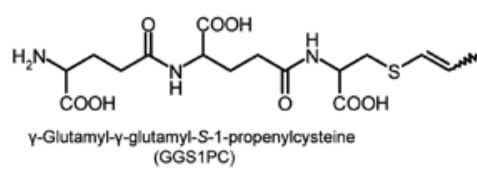<smiles>C=CCSCC(NC(=O)CCC(N)C(=O)O)C(=O)NCC(=O)O</smiles>

\section{D}<smiles>COc1cc(C2Oc3c(OC)cc(CCCO)cc3C2CO)ccc1O</smiles><smiles>COc1cc(C2Oc3c(OC)cc(/C=C/CO)cc3C2CO)ccc1O</smiles><smiles>CCCCCc1oc(OC)c(OC)c(=O)c1O</smiles>

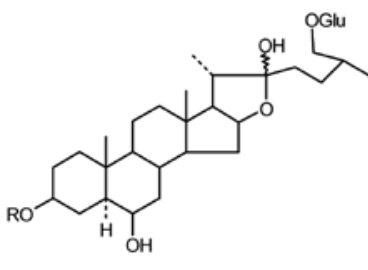

(DDC)<smiles>C=CCSSCC(NC(=O)CCC(N)C(=O)O)C(=O)NCC(=O)O</smiles>

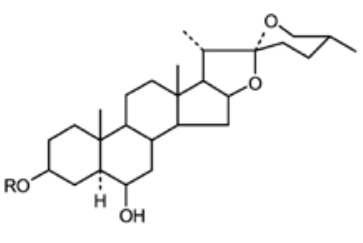

Spirostanol form saponin

Figure 1. Chemical structures of compounds found in garlic and aged garlic extract. Chemical structures of compounds in (A) raw garlic and (B-D) aged garlic extract. (B) Hydrophobic compounds, (C) $S$-alk(en)ylcysteine and $\gamma$-glutamylpeptide derivatives, and (D) phenolic compounds and saponins. 


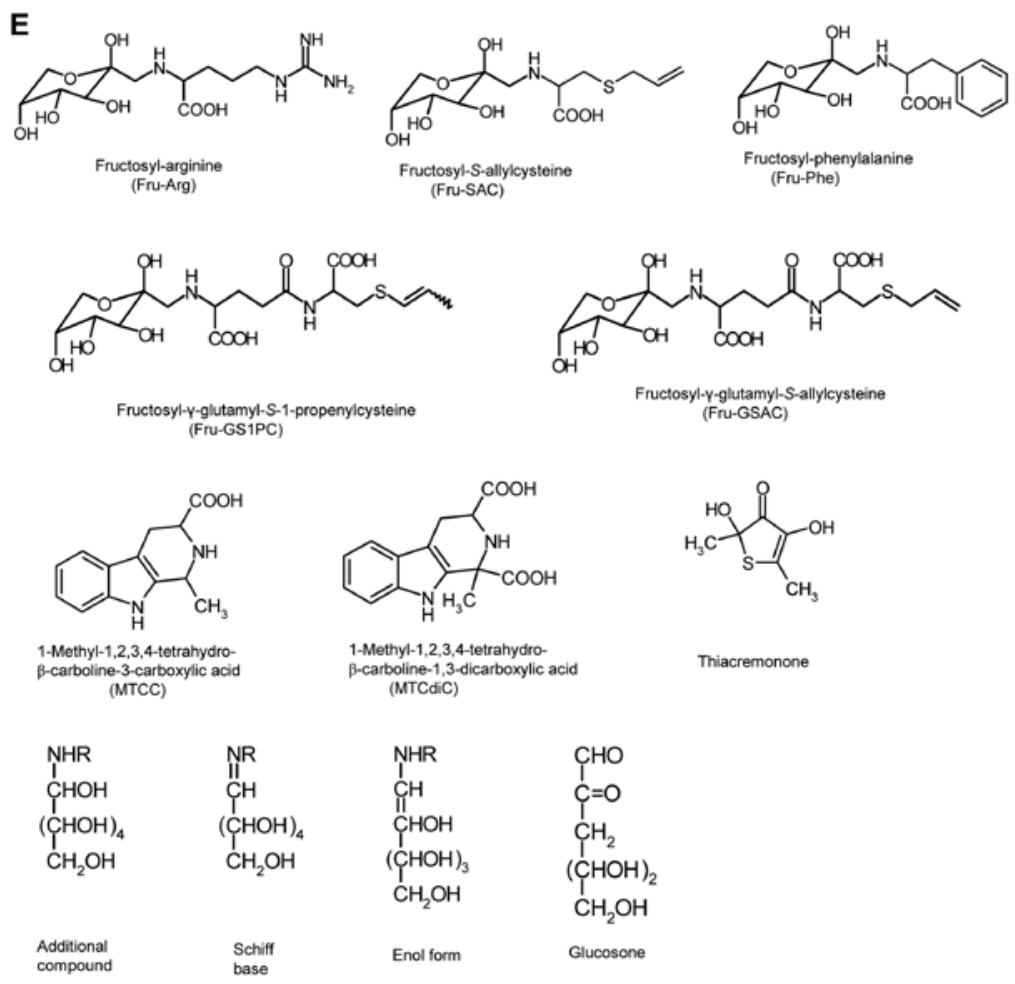

Figure 1. Continued. Chemical structures of compounds found in garlic and aged garlic extract. (E) Chemical structures of compounds in Maillard reaction-relating compounds.

known to be produced from GSAC by enzymatic reaction (Fig. 2C) (13). Garlic abundantly contains the trans-form of GS1PC, a plausible precursor compound of S1PC $(13,30,31)$. The content of cis-GS1PC in raw garlic has been shown to be $<50 \%$ of the cis-S1PC content in AGE on a molar basis, although the content of cis-S1PC gradually increases during the aging period $(30,31)$. The difference in content between the precursor and product suggests that cis-S1PC is produced not only from the precursor compound, $\gamma$-glutamyl peptide containing cis form of S1PC, but also from the other compounds $(30,31)$. The production mechanism of cis-S1PC was previously investigated with the hypothesis that 'cis-S1PC is produced via isomerization of trans-form' $(30,31)$. The model reaction to confirm the isomerization of S1PC was previously conducted using cis- and trans-form of S1PC organochemically synthesized on the chronological analysis of nuclear magnetic resonance (NMR) spectrum changes. While only one propenyl group form of signals was observed in each test sample at day 0 , both proton signals of cis- and trans-S1PC in the test substances at day 30 were detected with almost the same intensities (31). According to the results of chronological evaluation, the isomerization reaction was confirmed to occur reversibly (Fig. 2B). Divalent sulfides have an electron-releasing conjugative effect when unsaturated group or carbonium ion directly binds to sulfur $(32,33)$. This causes the cleavage of the $\pi$ bond between $\mathrm{C} 1$ and $\mathrm{C} 2$ in the unsaturated group. Although a part of cis-GS1PC in raw garlic was considered to be hydrolyzed by GGT to produce $c i s$-S1PC during the aging process, cis-GS1PC exhibits a slight increase rather than a sharp decline, similar to a trans-form (31). According to the results of S1PC isomerization experiments and the analysis of cis-GS1PC fluctuation during the aging process, some portion of $c i$-GS1PC in AGE also seems to be produced from trans-GS1PC. Although the biological properties of cis-S1PC have not yet been reported, the advances being made in garlic chemistry may soon precisely clarify these properties, including the associations between the biological activities and structure difference of cis- and trans-form. Furthermore, the balance of cis- and trans-form content may serve as a marker of the aging process, as the content of cis-form compound in fresh garlic is very small compared to that of trans-form; however, its amount increases with time.

\section{4. $\gamma$-Glutamyl- $\gamma$-glutamyl-S-alk(en)ylcysteines}

Raw garlic accumulates the dipeptides, $\gamma$-glutamyl- $S$-alk(en)ylcysteines as sulfur storage molecules that are precursor compounds of $S$-alk(en)ylcysteines/SACOs (e.g., SMC, SAC, S1PC, methiin, alliin and isoalliin) in the agricultural field $(1,6,13)$. The contents of these dipeptides in AGE decrease with the increasing $S$-alk(en)ylcysteine content (Fig. 2C) $(6,13,30,31)$. Nakamoto et al confirmed that the mass signal $[\mathrm{M}+\mathrm{H}]^{+}=420.1435$, which was likely tripeptides, $\gamma$-glutamyl- $\gamma$-glutamyl- $S$-allylcysteine (GGSAC) or $\gamma$-glutamyl- $\gamma$-glutamyl-cis/trans-S-1-propenylcysteine (cis/trans-GGS1PC), based on its elemental composition on the analysis of AGE (34). They isolated and identified the peak as the GGSAC using preparative high-performance liquid chromatography (HPLC), liquid chromatography/high resolution mass spectrometry (LC-HRMS) and NMR comparison with authentic chemically synthesized compounds. $\gamma$-Glutamyl- $\gamma$-glutamyl$S$-methylcysteine (GGSMC) and cis/trans-GGS1PC were isolated and identified in the same manner as GGSAC 


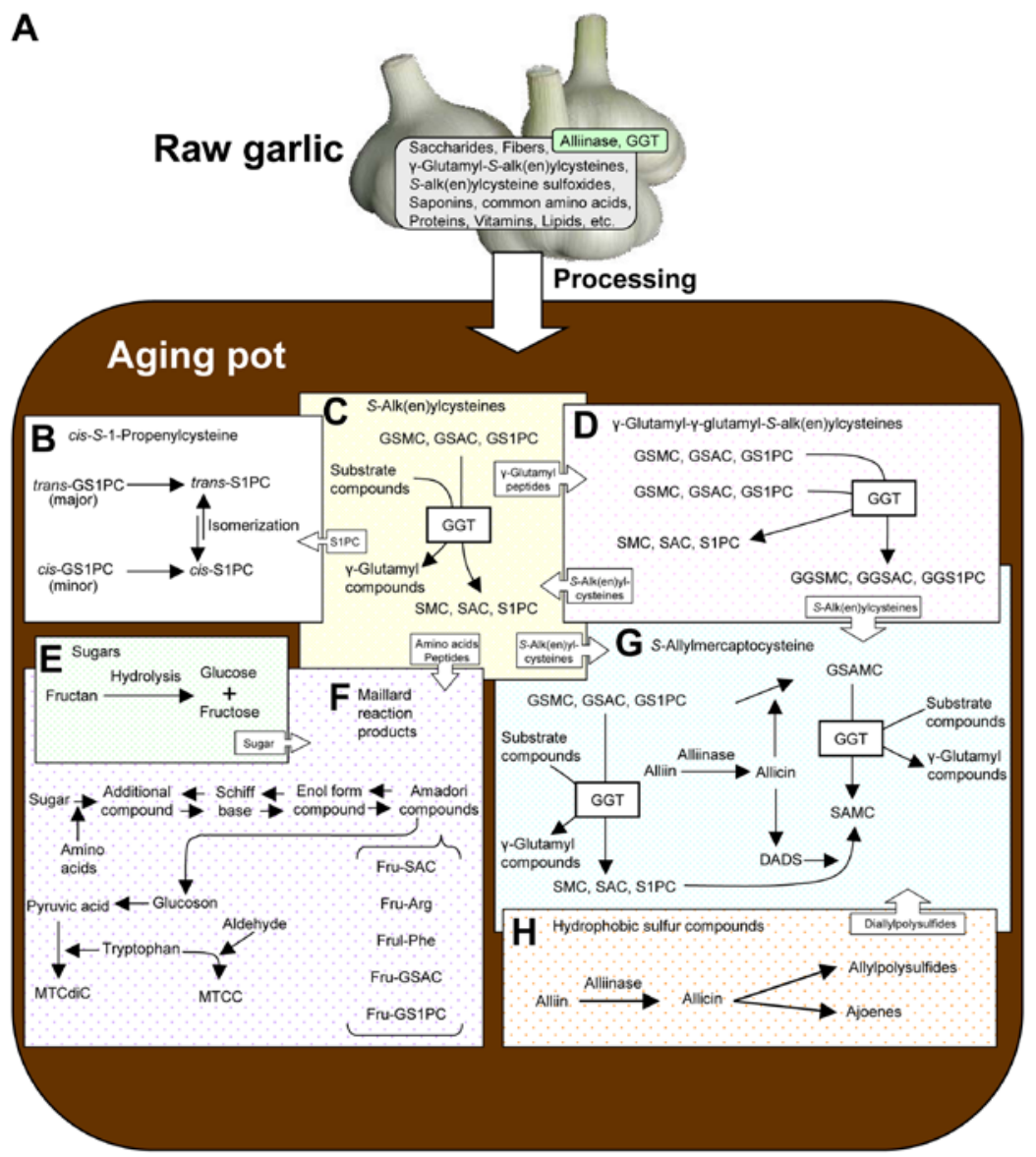

Figure 2. Constituents in raw garlic and production pathways of characteristic compounds in aged garlic extract. (A) Constituents in raw garlic. (B) Production mechanism of $c i s-S$-1-propenylcysteine by isomerization. (C) Production mechanism of $S$-alk(en)ylcyteine by enzymatic reaction and compounds produced. (D) Production mechanism of $\gamma$-glutamyl tripeptides by enzymatic reaction and compounds produced. (E) Hydrolysis of polysaccharide, fructan. (F) Production mechanism of Amadori compounds by the Maillard reaction and compounds produced. (G) Production mechanism of $S$-allylmercaptocysteine. (H) Hydrophobic compounds derived from alliin and their transformed compounds. Combination of block arrows and compound names indicate the providing of raw materials to the reaction pathways involved. Please refer to the corresponding names spelled out and the structures shown in Fig. 1 for abbreviated compound names in this figure. GGT, $\gamma$-glutamyl transpeptidase.

isolation/identification (34). Although GGSMC has been found in Vigna radiata seeds and Vigna mungo seeds (35), there are no reports available to date (at least to the best of our knowledge) of the identification of $\gamma$-glutamyl- $\gamma$-glutamyl tripeptides GGSMC in Allium plants, whereas other types of $\gamma$-glutamyl tripeptides, GSH and its derivatives, $S$-methylglutathione and $S$-( $\beta$-carboxypropyl)-glutathione, have been confirmed as intermediate compounds in the biosynthesis pathway of SACOs (Fig. 1C) $(6,13)$. Furthermore, the isolation and identification of GGSAC and cis/trans-GGS1PC were not previously reported (at least to the best of our knowledge) before the recently published study by Nakamoto et al (34).

Nakamoto et al hypothesized that $\gamma$-glutamyl- $\gamma$-glutamyl tripeptides were produced from two molecules of $\gamma$-glutamyl dipeptides by the endogenous GGT in garlic during the aging period, and conducted the model reactions using synthesized $\gamma$-glutamyl dipeptides and a protein fraction derived from garlic having GGT activity, which were inhibited by the GGT inhibitor (34). The production of $\gamma$-glutamyl- $\gamma$-glutamyl tripeptides was also confirmed in laboratory experiments mimicking the aging process, and GGT inhibitor suppressed the production of these compounds (Fig. 2D) (34). Both cis-GGS1PC and trans-GGS1PC were identified and the proportion of their content in AGE was approximately cis/trans $=10 / 90(\%)$ that was similar to the ratio of cis/trans-S1PC at aging at 16-22 months $(30,31)$. According to the above-mentioned report concerning the production mechanism of cis-S1PC in AGE, the cis form of GGS1PC can be produced from the trans form by an isomerization reaction during the aging process, as the contents of the cis-GS1PC in raw garlic are much lower than those in $\operatorname{AGE}(13,31,34)$. The study by Nakamoto et al suggested that $\gamma$-glutamyl tripeptides are produced via the transfer of $\gamma$-glutamyl- $S$-alk(en)ylcysteines to glutamic acid in other $\gamma$-glutamyl- $S$-alk(en)ylcysteines by an endogenous catalyst in garlic, which helps to simultaneously produce $\gamma$-glutamyl- $\gamma$-glutamyl- $S$-alk(en)ylcysteines and $S$-alk(en)ylcysteines during the aging process. Furthermore, the aging process in an aqueous alcoholic solution can provide the conditions under which to produce $\gamma$-glutamyl tripeptides that are formed by endogenous GGT in raw garlic.

To date, at least to the best of our knowledge, there is no report available concerning the biological properties of three $\gamma$-glutamyl tripeptides above. Kasai et al suggested that GGSMC is a useful index compound for the quality control of imported Vigna radiata seeds and Vigna mungo seeds (35). These three compounds may be the same as a marker/index compound for the aging process using an aqueous alcoholic 
solution, as the contents of these compounds in raw garlic are $<10-20 \%$ of the corresponding amount in AGE, since these compounds are generated mainly during the aging process (34).

\section{S-Allylmercaptocysteine}

SAMC is formed in AGE as a characteristic hydrophilic compound, and its biological properties have been evaluated $(13,19)$. However, this compound is not detected in fresh whole garlic, and only a trace amount is found when garlic is crushed (approximately $0.01 \mu \mathrm{mol} / \mathrm{g}$ fresh garlic) (13). SAMC is chemically synthesized by the reaction between allicin and Cys that proceeds very rapidly, even at room temperature $(13,36)$. Allicin is rapidly and abundantly produced from the precursor, alliin, when raw garlic is crushed or sliced; however, Cys is not detectable in whole raw garlic and crushed garlic (13). Fujii et al analyzed the chronological fluctuation of the SAMC content during the aging process and found that its content gradually increased during the aging process (37). Furthermore, allicin disappears in the early stage of the aging process. These findings indicate that SAMC in AGE can be produced without Cys during the aging process. This is different from the production mechanism in the textbook involving the reaction between allicin and Cys.

Matsutomo et al demonstrated that SAMC was produced by the reaction between SAC/S1PC and DADS using model reactions, and the amount of production was temperature-dependent (e.g., $25^{\circ} \mathrm{C}<<40^{\circ} \mathrm{C}<<60^{\circ} \mathrm{C}$ ) (30). Since the aging process normally occurs at room temperature, the contribution of the reaction involving DADS/SAC/S1PC towards total SAMC production may be minimal (Fig. 2G).

Matsutomo et al also demonstrated the presence of $\gamma$-glutamyl- $S$-allylmercaptocysteine (GSAMC) in AGE by LC-HRMS analysis, a putative precursor of SAMC, similar to GSAC for a precursor of SAC and GS1PC for a precursor of S1PC (Fig. 1C). GSAMC is not detectable in raw garlic (30), although its content in AGE reaches a maximum level within one month and then gradually decreases (37). Based on these fluctuations of GSAMC and SAMC in AGE, Fujii et al hypothesized that GSAMC is produced from GSAC/GS1PC in the early stages of the aging process, and that SAMC is produced from GSAMC by endogenous GGT as an alternative mechanism of SAMC production (37). They conducted model reactions and laboratory experiments mimicking the aging process using GSAC, GS1PC, alliin, allicin, garlic protein fraction (GPF) having GGT activity and raw garlic cloves (37). The production of GSAMC and SAMC was observed in the combination of GS1PC/allicin/GPF and GSAC/allicin/GPF, and SAMC production was inhibited by GGT inhibitor. GGT inhibitor also suppressed SAMC production in laboratory experiments mimicking the aging process using raw garlic.

According to the above-mentioned observations, the production of SAMC during the aging process is postulated as follows: First, when garlic is soaked in an aqueous alcoholic solution, alliin is transformed to allicin by alliinase, and GSAMC is produced by the reaction between allicin and GSAC/GS1PC, and finally SAMC is produced from GSAMC by GGT (Fig. 2G). Thus, it is suggested that GSAMC and
SAMC in AGE are produced through the combination of chemical and enzymatic reactions during the aging process. The two production pathways of SAMC mentioned above differ from the ones in the textbook, and suggest that SAMC can be made without endogenous Cys during aging process. Furthermore, SAMC production involves plural reaction pathways that provide several raw materials by the chemical and enzymatic reactions. This is not a simple reaction, such as the condensation of two raw materials, Cys and allicin.

SAMC has been reported to possess useful biological properties, such as hepatoprotective effects, anti-ototoxicity and anticancer effects (38-41). However, SAMC quickly disappeared when it was mixed with blood (26). Recently, Yang et al reported the metabolism of SAMC using LC-MS/MS, and revealed that its half-life in rat plasma was $<5 \mathrm{~min}$ and its clearance was within $30 \mathrm{~min}$ (42). These results suggest that SAMC has strong affinity to proteins in plasma or that the metabolite of SAMC may be an active molecule.

\section{Hydrophobic sulfur compounds}

Following the discovery of allicin and the alliin-allinase system in the early 1940s, the scientific knowledge in the garlic chemistry, particularly that relating to the chemical and biological properties of hydrophobic compounds, has rapidly expanded (7,43). Allicin has always played a central role in garlic chemistry; however, this compound has chemical characteristics of low stability and high reactivity $(26,27)$. Allicin transforms into various sulfur compounds, such as DASn, ajoenes and vinyldithiins (Figs. 1B and 2H) (7). Transformation products have also been widely investigated in terms of their biological and chemical properties by several researchers $(13,43)$. These include characteristic constituents in the lipophilic products, such as garlic oil and oil macerate product. AGE is characterized by water-soluble constituents and its content of lipophilic substances is very low $(7,10)$. Among these hydrophobic compounds in AGE, DASn are main lipophilic constituents and play an important role in producing one of the characteristic sulfur compounds, SAMC. Matsutomo et al performed model reactions to confirm the production mechanism of SAMC using DADS, SAC and S1PC (Fig. 2G and H) (30). The production of SAMC was observed in the model reaction mixtures of DADS/SAC and DADS/S1PC, and its production was enhanced in a temperature-dependent manner. These results indicate that some hydrophobic volatile compounds in AGE are condensed into a hydrophilic compound through the production of SAMC by the reaction with $\mathrm{SAC} / \mathrm{S} 1 \mathrm{PC}$ during the aging process. The appropriate balance of hydrophilicity and hydrophobicity in AGE may provide the proper condition for the production of SAMC.

\section{Maillard reaction products}

The amino-carbonyl reaction, namely, the Maillard reaction, is known as a chemical reaction between reducing sugars and the amino group in amino acids and peptides. This reaction occurs non-enzymatically and yields browning, characteristic flavors and tastes to food materials (44). Although raw garlic contains abundant polysaccharides and amino acids, the contents of 
reducing monosaccharides, such as Glc, galactose and Fru, are very low (Fig. 2E and F). Ryu et al isolated and identified Fru-Arg as a Maillard reaction product in AGE that was produced by the reaction between Glc and Arg (Fig. 1E) (11). Arg is the most abundant common amino acid in raw garlic, although free Glc is not detectable. Although Fru-Arg is not detectable within four months of aging, its content gradually increases with the further progression of aging, as Glc is supplied into the container in which the aging process occurs by the degradation of fructan, having Glc at the reducing end (please see section 8 below entitled 'Sugars'). Matsutomo et al isolated and identified the 'Fru-Arg' type of sulfur-containing Amadori compounds, such as $N \alpha$-(1-deoxy-D-fructos-1-yl)-Sallylcysteine (Fru-SAC), N $\alpha$-(1-deoxy-D-fructos-1-yl)- $\gamma$-glutamyl- $S$-allylcysteine (Fru-GSAC) and $N \alpha$-(1-deoxy-D-fructos -1-yl)- $\gamma$-glutamyl-S-1-propenyl-cysteine (Fru-GS1PC) in AGE (Fig. 1E) (45). These sulfur-containing Amadori compounds are characteristic compounds in AGE as they are produced during the aging process from GSAC and GS1PC that are characteristic constituents in A. sativum $\mathrm{L}$.

Ide et al reported the identification of four Maillard reaction products,namely (1R,3S)-1-methyl-1,2,3,4-tetrahydro- $\beta$-carboline-3-carboxylic acid [(1R, 3S)-MTCC], (1S, 3S)-1-methyl-1, 2, 3,4-tetrahydro- $\beta$-carboline-3-carboxylic acid [(1S,3S)-MTCC], (1R, 3S)-1-methyl-1, 2, 3, 4-tetrahydro- $\beta$-carboline-1, 3-dicarboxylic acid [(1R, 3S)-MTCdiC], (1S, 3S)-1-methyl-1, 2, 3, 4-tetrahydro- $\beta$-carboline-1, 3-dicarboxylic acid [(1S, 3S)-MTCdiC] that were produced by the reaction between tryptophan and acetaldehyde/pyruvic acid (Figs. 1E and 2F) (46). These compounds differ from the type of 'Fru-Arg'. Small amounts of these four compounds were found in four month-aging material, and their contents were then gradually increased. Raw garlic contains tryptophan, while acetaldehyde and pyruvic acid are not detectable. Acetaldehyde may be produced from ethanol added and pyruvic acid is generated by the degradation of alliin and deoxyglucoson produced by the Maillard reaction (46). When the aging process begins, the degradation of alliin immediately occurs, and the production/degradation of deoxyglucoson progresses later than the degradation of alliin. Therefore, these four compounds may be generated earlier than Fru-Arg production, while the degradation of polysaccharides and the production/degradation of deoxyglucoson are also necessary for providing pyruvic acid.

Reactive oxygen species (ROS) play an important role in the induction of various diseases. AGE has been shown to have antioxidant activities, including the scavenging of ROS, and its sulfur-containing constituents are mainly responsible for these antioxidant activities (47). Ryu et al and Ide et al evaluated the antioxidant activity of their isolated Maillard reaction products by $\mathrm{H}_{2} \mathrm{O}_{2}$ scavenging activity, and found that the antioxidant activity of (1S,3S)-MTCdiC was particularly more potent than that of ascorbic acid $(11,46)$. Zhou et al reported that Fru-Arg attenuated neuroinflammatory responses and Nrf2-mediated oxidative stress response in microglial cells (48). Oxidative stress in neuron cells induces brain dysfunction, resulting in severe symptoms, such as cognitive impairment and Alzheimer's disease. These results indicate that the aging process generates the various antioxidant compounds via the Maillard reaction.

\section{Sugars}

Carbohydrates are the most abundant components in raw garlic, which are approximately $80 \%$ of the dry weight $(8,49)$. Among these, the main constituent is a water-soluble fructo-polysaccharide termed fructan, which has the structure of an inulin type $2 \rightarrow 1$ linkage and Glc molecule at the reducing end $(13,49)$. Fructan is the main carbohydrate storage compound and may play a role in osmotic regulation and in cold resistance (50). Its amount in raw garlic is $>60 \%$ of the dry weight (49). The other carbohydrate constituents are the monosaccharides, Glc and Fru, disaccharide sucrose and the polysaccharides, galactan, galacturonan, xyloglucan and pectin $(13,51)$. Fructan is gradually hydrolyzed into the monosaccharides, Fru and Glc, during the aging process (Fig. 2E). The Glc content in AGE has been shown to be 3.3\%, indicating that a number of fructans remained without hydrolysis (11). On the other hand, black garlic is produced by continuous heating at $70-80^{\circ} \mathrm{C}$ under high humidity conditions for several months; subsequently, fructan is completely hydrolyzed into monosaccharide, and the resulting product becomes sweet, wet and sticky $(8,9,52)$. The contents/balance of monosaccharides and polysaccharides affects the development of the product formulation through changes in physical properties, such as viscosity and density in liquid form, promoting the dissolution of slightly soluble substances in liquid form, the binding ability of the powder in solid form, the active water content in solid form, and so on. Furthermore, the monosaccharide Fru content affects the taste in the final product, which contributes to the sweetness.

It is well known that sugars react with amino acids to produce the Maillard reaction products (44). Although the amount of reducing monosaccharides is low in raw garlic, its content during the aging process gradually increases with the progression of the aging process, and the content of Maillard reaction products fluctuates in a synchronized manner (please see the section 7 above entitled 'Maillard reaction products'). Fructans and the degradation products of fructans may characterize AGE both physically and chemically.

\section{Saponins}

Saponin is a general term for compounds in natural products that produce foam and possess surface-active properties as they consist of a fat-soluble aglycone linked to one or more water-soluble carbohydrate chains. A number of plants contain saponins, and in particular, ginsenoside in ginseng and glycyrrhizin in liquorice are well known as useful saponins for human health. In their chemical structures, they are divided into two groups, triterpenoid saponins and steroidal saponins. Furthermore, steroidal saponins are divided into two forms, namely spirostanol and furostanol. Although Smoczkiewicz et al suggested in the early of 1980s that garlic contains saponins having a structure of steroidal glycoside, the isolation and structural determination of these had not yet been accomplished (53). Towards the end of the 1980s, Matsuura et al successfully isolated novel furostanol glycosides termed proto-eruboside-B and satiboside-B from a crude glycoside fraction that was prepared from methanol extract of frozen garlic (Fig. 1D) $(54,55)$. Furthermore, they found that 
the combination of freezing and extraction with methanol was effective by the suppression of glucosidase activity in order to isolate genuine glycosides from raw materials. That is, no spirostanol glycosides were observed in the extract with the combination treatment of freezing and extraction with methanol. However, spirostanol glycosides were observed in the methanol extract of garlic without freezing treatment.

In the steroidal saponins isolated from garlic, spirostanol types have several biological activities and effects, such as antimicrobial activities (54), anticancer activities (56), inhibitory effects on blood coagulability (57), promoting effects on fibrinolysis (57) and cholesterol-lowering effects (58). However, genuine glycosides furostanol types do not have these activities. According to previous studies (54-58), the processing method involving the reaction with endogenous enzymes in garlic may be crucial for the production of the useful biological activities.

A. ampeloprasum L. (common name: Elephant garlic) is often used as garlic. This plant is commonly known as wild leek, while its appearance is very similar to A. sativum L. in its stalk, leaves, cloves and bulb, apart from the size of the clove and bulb. A. ampeloprasum L. also contains spirostanol glycoside having agigenin as an aglycone (56). On the other hand, the sapogenin part of spirostanol glycoside derived from garlic is $\beta$-chlorogenin. Itakura et al successfully established the convenient analytical method of TLC to distinguish A. sativum L. and A. ampeloprasum L. using the difference of aglycones in their saponins (59). Furthermore, their method can distinguish A. sativum L. from other Allium plants, such as A. cepa (onion), A. porrum (leek), A. fistulosum (Welsh onion), and so on, even they are processed into commercial products $(56,59)$.

\section{Compounds of which the production mechanisms have not been elucidated}

The existence of two sulfanyl-alanines, 3-allyltrisulfanyl-alanine (3ATSA) and 4-allyltetrasulfanyl-alanine (4ATSA), was confirmed by the LC-HRMS analysis of AGE: 3ATSA, calculated $[\mathrm{M}+\mathrm{H}]^{+}=226.0025$, observed $[\mathrm{M}+\mathrm{H}]^{+}=226.0021 ;$ 4ATSA, calculated $[\mathrm{M}+\mathrm{H}]^{+}=257.9745$, observed $[\mathrm{M}+\mathrm{H}]^{+}=257.9744$ (Fig. 1C). 3ATSA has been reported as a novel sulfur compound derived from garlic having a potent anti-yeast activity, which is generated when garlic is heated at $120^{\circ} \mathrm{C}$ for $30 \mathrm{~min}$ and completely disappears following $90 \mathrm{~min}$ of heating, while its content in heated garlic and production mechanisms have not been described (60). The MS signal of 3ATSA is not detectable in 4-month-old AGE; however, its content gradually increases in the subsequent aging process, while the aging process occurs at room temperature. Although the content of 4ATSA is lower than that of 3ATSA, it gradually increases with aging. According to the fluctuation during the aging process, it may be produced by the reaction between $S$-alkenylcysteines and DASn, such as SAMC production mechanisms (please see sections 5 and 6 above, entitled ' $S$-Allylmercaptocysteine' and 'Hydrophobic sulfur compounds', respectively).

Two GSH derivatives, $S$-allylglutathione (SAG) and $S$-allylmercaptoglutathione (SAMG), were also confirmed by the LC-HRMS analysis of AGE, yielding mass signals of theoretical elemental composition of each compound: SAG, calculated
$[\mathrm{M}+\mathrm{H}]^{+}=348.1224$, observed $[\mathrm{M}+\mathrm{H}]^{+}=348.1219 ; \mathrm{SAMG}$, calculated $[\mathrm{M}+\mathrm{H}]^{+}=380.0945$, observed $[\mathrm{M}+\mathrm{H}]^{+}=380.0940$ (Fig. 1C). SAG is thought to exist in raw garlic as an intermediate on alliin synthesis pathway involving $S$-alk(en)ylation of Cys residue, deglycinylation of GSH, $S$-oxygenation of SAC moiety and deglutamylation of $\gamma$-glutamylpeptide, while its source in the allyl group is unknown (13). SAG in AGE may be the remaining part not being used for alliin production. Rabinkov et al charactrerized the chemical and biological properties of SAMG that was organochemically synthesized using allicin and GSH (61). To date, to the best of our knowledge, there is no report available to indicate that raw garlic contains SAMG. $S$-Allylsulfanylation on GSH/SAG could occur similar to SAMC production; however, the production mechanisms of SAMG are unknown.

Ban et al reported the induction of colon cancer apoptosis by a unique heterocyclic compound, thiacremonone, derived from heated garlic (Fig. 1E) (62). This compound is known to be related to pigment and aroma in soy sauce, miso, beer, heated garlic and heated onion, which is produced by the Maillard reaction between Cys/cystine (Cys2) and Fru (63). This compound in AGE has been investigated by LC/LC-MS analysis and its content has been found to be lower than a few micrograms per dry-material base of AGE. Fru is abundantly produced by hydrolysis of fructan during the aging process, while the small amount is present in raw garlic (please see section 8 above entitled 'Sugars'). Since Cys and Cys2 are not detectable in raw garlic (13); thiacremonone in AGE could be produced by the Maillard reaction between Fru and some of Cys derivatives, such as SAC, S1PC and SAMC. However, at the present time, its production mechanism during the aging process are unclear.

Matsutomo et al isolated and identified coniferyl alcohols and dilignol drivatives, (-)-(2R,3S)-dihydrodehydrodiconiferyl alcohol (DDDC), (+)-(2S,3R)-dehydrodiconiferyl alcohol (DDC), ery thro-guaiacylglycerol- $\beta-\mathrm{O}-4$ '-coniferyl ether and threo-guaiacylglycerol- $\beta$-O-4'-coniferyl ether in AGE by HPLC under the activity-guided fractionation of antioxidant activity (Fig. 1D) (12). These compounds exhibit a high antioxidant activity. Yamakawa et al examined the effects of DDDC and DDC on primary human coronary artery smooth muscle cells, and only DDC inhibited alkaline phosphatase activity in cells (64). The production mechanisms of these phenolic compounds are unclear. Ichikawa et al isolated and identified six phenolic compounds, namely trans-coumaric acid,trans-ferulic acid, $\mathrm{N}$-trans-coumaroyloctopamine, $\mathrm{N}$-trans-feruloyloctopamine, guaiacylglycerol- $\beta$-ferulic acid and guaiacylglycerol- $\beta$-caffeic acid methyl ether, as an antioxidant derived from garlic skin (65). According to their report, DDC and DDDC may be degradation products of lignans in garlic skin or cell walls.

Kodera et al isolated and identified a unique phenolic compound, 3-hydroxy-5-methoxy-6-methyl-2-n-pentyl-4H -pyran-4-one, named allixin (Fig. 1D) (66). This compound is classified as phytoalexin that is produced by plants when they are subjected to stress from their environment, and is the first phytoalexin isolated from garlic (66-68). This compound exhibits weak antimicrobial activity (66), potent antitumor activity in vivo/in vitro (69) and neurotrophic activity (70). Phytoalexins are synthesized de novo by plants at harmed areas by pathogen infection, or physical/chemical stress. Kodera et al induced this compound using several phys- 
ical/chemical stresses; however, the details of the biosynthesis of this compound are not clear (66-68). Allixin in AGE may be produced before the aging process or at the very early period of aging.

\section{Conclusion}

The constituents in raw garlic can change into various compounds depending on the processing method and conditions. The aging process produces both hydrophilic and hydrophobic substances by the enzymatic and chemical reactions, including transformation, hydrolysis, isomerization, the Maillard reaction, etc. During the aging process, some hydrophobic volatile compounds reacted with hydrophilic compounds to produce useful constituents in AGE, indicating that the aging process can condense even volatile compounds and these reactions lead to a greater diversity of constituents in AGE. Aging is a superior processing method as some of the constituents in AGE have been found to possess useful biological properties which are beneficial to human health.

\section{Acknowledgements}

The authors would like to express their deep gratitude to Dr Takami Oka of Wakunaga Pharmaceutical Co., Ltd., for his helpful advice, encouragement and critical reading of the manuscript.

\section{Funding}

No funding was received.

\section{Availability of data and materials}

Not applicable.

\section{Authors' contributions}

YK designed the study, researched the literature, performed the analysis of the data in sections $2,3,4,5$ and 10, and drafted the manuscript. MK and MN also designed the study, researched the literature, performed the analysis of the data in sections 5 and 6 , and drafted the manuscript. TM also designed the study, researched the literature, performed the analysis of the data in sections $3,4,5$ and 10 , and drafted the manuscript. All author have reviewed and approved the final manuscript.

\section{Ethics approval and consent to participate}

Not applicable.

\section{Patient consent for publication}

Not applicable.

\section{Competing interests}

The authors declare that they have no competing interests.

\section{References}

1. Lancaster JE and Show ML: $\gamma$-Glutamyl peptides in the biosynthesis of $S$-alk(en)yl-L-cysteine sulphoxides (flavor precursors) in Allium. Phytochemistry 28: 455-460, 1989.

2. Yoshimoto N, Yabe A, Sugino Y, Murakami S, Sai-Ngam N, Sumi S, Tsuneyoshi T and Saito K: Garlic $\gamma$-glutamyl transpeptidases that catalyze deglutamylation of biosynthetic intermediate of alliin. Front Plant Sci 5: 758, 2015.

3. Yoshimoto N, Onuma M, Mizuno S, Sugino Y, Nakabayashi R, Imai S, Tsuneyoshi T, Sumi S and Saito K: Identification of a flavin-containing $S$-oxygenating monooxygenase involved in alliin biosynthesis in garlic. Plant J 83: 941-951, 2015.

4. Matsuura H, Inagaki M, Maeshiga K, Ide N, Kajimura Y and Itakura Y: Changes in contents of $\gamma$-glutamyl peptides and fructan during growth of Allium sativum. Platna Med 62: 70-71, 1996

5. Block E: The chemistry of garlic and onions. Sci Am 252: 114-119, 1985.

6. Kodera Y, Ushijima M, Amano H, Suzuki JI and Matsutomo T: Chemical and Biological Properties of S-1-Propenyl-L-Cysteine in Aged Garlic Extract. Molecules 22: E570, 2017.

7. Block E: The organosulfur chemistry of the genus Allium-implication for organic sulfur chemistry. Angew Chem Int Ed Engl 31: 1135-1178, 1992.

8. Kimura S, Tung YC, Pan MH, Su NW, Lai YJ and Cheng KC: Black garlic: A critical review of its production, bioactivity and application. Yao Wu Shi Pin Fen Xi 25: 62-70, 2017.

9. Martínez-Casas L, Lage-Yusty M and López-Hernández J: Changes in aromatic profile, sugars and bioactive compounds when purple garlic is transformed into black garlic. J Agric Food Chem 65: 10804-10811, 2017.

10. Kodera Y, Matsuura H, Sumiyoshi H and Sumi SI: Chapter 30. Garlic Chemistry: chemical and biological properties of sulfurcontaining compounds derived from garlic. In: ACS symposium series 851, Food Factors in Health Promotion and Disease Prevention. Shahadi F, Ho CT, Watanabe S and Osawa T (eds). American Chemical Society, Washington DC, pp346-357, 2003.

11. Ryu K, Ide N, Matsuura H and Itakura I: $N \alpha$-(1-deoxy-D-fructos1-yl)-L-arginine, an antioxidant compound identified in aged garlic extract. J Nutr 136 (Suppl 3): 972S-976S, 2006.

12. Matsutomo T, Stark TD and Hofmann T: In vitro activity-guided identification of antioxidants in aged garlic extract. J Agric Food Chem 61: 3059-3067, 2013.

13. Koch HP and Lawson LD (eds): Garlic: The science and therapeutic application of Allium Sativum L and related species. Williams \& Wilkins, 1996.

14. Ichikawa $\mathrm{M}$, Ide $\mathrm{N}$ and Ono $\mathrm{K}$ : Changes in organosulfur compounds in garlic cloves during storage. J Agric Food Chem 54: 4849-4854, 2006.

15. Sun Y, Hu J, Wang W, Zhang B and Shen Y: Characterization of $\gamma$-glutamyltranspeptidases from dormant garlic and onion bulbs. Food Sci Nutr 7: 499-505, 2019.

16. Amano H, Kazamori D, Itoh K and Kodera Y: Metabolism, excretion, and pharmacokinetics of $S$-allyl-L-cysteine in rats and dogs. Drug Metab Dispos 43: 749-755, 2015.

17. Amano H, Kazamori D and Itoh K: Pharmacokinetics and $N$-acetylation metabolism of $S$-methyl-L-cysteine and trans$S$-1-propenyl-L-cysteine in rats and dogs. Xenobiotica 46: 1017-1025, 2016.

18. Amano H, Kazamori D and Itoh K: Evaluation of the effects of $S$-allyl-L-cysteine, $S$-methyl-L-cysteine, trans$S$-1-propenyl-L-cysteine, amd their $N$-acetylated and $S$-oxidized metabolites on human CYP activities. Biol Pharm Bull 39: 1701-1707, 2016.

19. Amagase $\mathrm{H}$ : Clarifying the real bioactive constituents of garlic. J Nutr 136 (Suppl 3): 716S-725S, 2006.

20. Colín-González AL, Santana RA, Silva-Islas CA, ChánezCárdenas ME, Santamaría A and Maldonado PD: The antioxidant mechanisms underlying the aged garlic extract- and $S$-allylcysteine-induced protection. Oxid Med Cell Longev 2012: 907162, 2012

21. Fukushima S, Takada N, Wanibuchi H, Hori T, Min W and Ogawa M: Suppression of chemical carcinogenesis by watersoluble organosulfur compounds. J Nutr 136 (Suppl 3): 1049S-1053S, 2006.

22. Suzuki J, Yamaguchi T, Natsutomo T, Amano H, Morihara N and Kodera Y: S-1-Propenylcysteine promotes the differentiation of $\mathrm{B}$ cell into IgA-producing cells by the induction of Erk1/2-dependent Xbp1 expression in Peyer's patches. Nutrition 32: 884-889, 2016. 
23. Suzuki JI, Kodera Y, Miki S, Ushijima M, Takashima M, Matsutomo T and Morihara N: Anti-inflammatory action of cysteine derivative $S$-1-propenylcysteine by inducing MyD88 degradation. Sci Rep 8: 14148, 2018

24. Matsutomo T, Ushijima M, Kodera Y, Nakamoto M, Takashima M, Morihara N and Tamura K: Metabolomic study on the antihypertensive effect of $S$-1-propenylcysteine in spontaneously hypertensive rats using liquid chromatography coupled with quadrupole-Orbitrap mass spectrometry. J Chromatogr B Analyt Technol Biomed Life Sci 1046: 147-155, 2017.

25. Pushpendran CK, Devasagayam TP, Chintalwar GJ, Banerji A and Eapen J: The metabolic fate of [35S]-diallyl disulphide in mice. Experientia 36: 1000-1001, 1980

26. Lawson LD and Wang ZJ: Pre-hepatic fate of the organosulfur compounds derived from garlic (Allim sativum). Planta Med 59: 688A-689A, 1993.

27. Freeman F and Kodera Y: Garlic chemistry: Stability of $S$-(2-propyl) 2-propen-1-sulfinothioate (allicin) in blood, solvents, and stimulated physiological fluids. J Agric Food Chem 43: 2332-2338, 1995

28. Amagase H, Petesch BL, Matsuura H, Kasuga S and Itakura Y: Intake of garlic and its bioactive components. J Nutr 131 (Suppl 3): 955S-962S, 2001

29. Sugii M, Suzuki T and Nagasawa S: Isolation of (-)S-propenyl-Lcysteiene from garlic. Chem Abstr 59: 6509, 1963.

30. Matsutomo T and Kodera Y: Development of an analytical method for sulfur compounds in aged garlic extract with the use of a post-column high performance liquid chromatography method with sulfur-specific detection. J Nutr 146: 450S-455S, 2016.

31. Kodera Y, Matsutomo T and Itoh K: The evidence for the production mechanism of $c i s-S$-1-propenylcysteine in aged garlic extract based on a model reaction approach using its isomers and deuterated solvents. Planta Med Lett 2: e69-e72, 2015.

32. Oae S, Tamagaki S and Kunieda N: Stereoelectronic effect of sulfur group. In: Organic Sulfur Chemistry. Oae S (ed) Kagakudojin, Kyoto, pp41-81, 1982.

33. Oae S, Ohno A and Tagaki W: Acid-catalyzed hydrogen-deuterium exchange reaction of deuterated anisole, thioanisole and benzene. Bull Chem Soc Jpn 35: 681-683, 1962.

34. Nakamoto M, Fujii T, Matsutomo T and Kodera Y: Isolation and identification of three $\gamma$-glutamyl tripeptides and their putative production mechanism in aged garlic extract. J Agric Food Chem 66: 2891-2899, 2018

35. Kasai T, Shiroshita Y and Sakamura S: $\gamma$-Glutamyl peptides of Vigna radiata seeds. Phytochemistry 25: 679-682, 1986.

36. Albrecht F, Leontiev R, Jacob C and Slusarenko AJ: An optimized facile procedure to synthesize and purify allicin. Molecules 22: $770,2017$.

37. Fujii T, Matsutomo T and Kodera Y: Changes of $S$-allylmercaptocysteine and $\gamma$-glutamyl-S-allylmercaptocysteine contents and their putative production mechanisms in garlic extract during the aging process. J Agric Food Chem 66: 10506-10512, 2018.

38. Sumioka I, Matsura T and Yamada K: Therapeutic effect of $S$-allylmercaptocysteine on acetaminophen-induced liver injury in mice. Eur J Pharmacol 433: 177-185, 2001.

39. Xiao J, Guo R, Fung ML, Liong EC, Chang RC, Ching YP and Tipoe GL: Garlic-derived $S$-allylmercaptocysteine ameliorates nonalcoholic fatty liver disease in a rat model through inhibition of apoptosis and enhancing autophagy. Evid Based Complement Alternat Med 2013: 642920, 2013.

40. Uzun L, Kokten N, Cam OH, Kalcioglu MT, Ugur MB, Tekin $M$ and Acar GO: The effect of garlic derivatives ( $S$-allylmercaptocysteine, diallyl disulfide, and $S$-allylcysteine) on gentamicin induced ototoxicity: An experimental study. Clin Exp Otorhinolaryngol 9: 309-313, 2016.

41. Zhu X, Jiang X, Li A, Sun Y, Liu Y, Sun X, Feng X, Li S and Zhao Z: $S$-Allylmercaptocysteine suppresses the growth of human gastric cancer xenografts through induction of apoptosis and regulation of MAPK and PI3K/Akt signaling pathways. Biochem Biophys Res Commun 491: 821-826, 2017.

42. Yang M, Dong Z, Jiang X, Zhao Z, Zhang J, Cao X and Zhang D: Determination of $S$-allylmercaptocysteine in rat plasma by LC-MS/MS and its application to a pharmacokinetics study. J Chromatogr Sci 56: 396-402, 2018.

43. Block E: Garlic and Other Alliums: The Lore and the Science. The Royal Society of Chemistry, Cambridge, 2010.

44. Rizzi GP: The Maillard Reaction in Foods. In: Maillard Reactions in Chemistry, Food, and Health. Labuza TP, Reineccius GA, Monnier VM and Baynes JW (eds). The Royal Society of Chemistry, Cambridge, pp11-19, 1994.
45. Matsutomo T, Stark TD and Hofmann T: Targeted screening and quantitative analyses of antioxidant compounds in aged-garlic extract. Eur Food Res Technol 244: 1803-1814, 2018.

46. Ide N, Ichikawa M, Ryu K, Yoshida J, Sasaoka T, Sumi S and Sumiyoshi H: Chapter 22. Antioxidants in processed garlic: Tetrahydro- $\beta$-carboline derivatives in aged garlic extract. In: ACS Symposium Series 851. Food Factors in Health Promotion and Disease Prevention. Shahidi F, Ho CT. Watanabe S and Osawa T (eds). American Chemical Society, Washington, DC, pp250-263, 2003.

47. Imai J, Ide $\mathrm{N}$, Nagae $\mathrm{S}$, Moriguchi $\mathrm{T}$, Matsuura $\mathrm{H}$ and Itakura $\mathrm{Y}$ : Antioxidant and radical scavenging effects of aged garlic extract and its constituents. Planta Med 60: 417-420, 1994.

48. Zhou H, Qu Z, Mossine VV, Nknolise DL, Li J, Chen Z, Cheng J, Greenlief CM, Mawhinney TP, Brown PN, et al: Proteomic analysis of the effects of aged garlic extract and its FruArg component on lipopolysaccharide-induced neuroinflammatory response in microglial cells. PLoS One 9: e113531, 2014.

49. Fenwick TE and Hanley AB: Critical review in food science and nutrition. In: The genus Allium. Part 2. Furia TE (ed). CRC Press, Boca Raton, FL, p284, 1985.

50. Darbyshire B and Henry RJ: Differences in fructan content and synthesis in some Allium species. New Phytol 87: 249-256, 1981.

51. Ohsumi $\mathrm{C}$ and Hayashi T: The oligosaccharide unit of xyloglucan in the cell wall of bulbs of onion, garlic, and their hybrid. Plant Cell Physiol 35: 963-967, 1994.

52. Kang OJ: Physicochemical Characteristics of Black Garlic after Different Thermal Processing Steps. Prev Nutr Food Sci 21: 348-354, 2016.

53. Smoczkiewicz MA, Nitschke D and Wieladek H: Microdetermination of steroid and triterpene saponin glycosides in various plant materials I. Allium species. Mikrochim Acta 78: 43-53, 1982

54. Matsuura H, Ushiroguchi T, Itakura $\mathrm{Y}$, Hayashi $\mathrm{N}$ and Fuwa T: A frostanol glycoside from garlic, bulbs of Allium sativum $\mathrm{L}$. Chem Pharm Bull (Tokyo) 36: 3659-3663, 1988.

55. Matsuura H, Ushiroguchi T, Itakura Y and Fuwa T: Further studies on steroidal glycosides from bulbs, root and leaves of Allium sativum L. Chem Pharm Bull (Tokyo) 37: 2741-2743, 1989.

56. Matsuura H: Phytochemistry of garlic horticultural and processing procedures. In: Neutraceuticals: Designer Foods III. Garlic, Soy and Licorice. Lachance PA (ed). Food and Nutrition Press, Trumbull, CT, pp55-69, 1997.

57. Peng JP, Chen H, Qiao YQ, Ma LR, Narui T, Suzuki H, Okuyama $\mathrm{T}$ and Kobayashi H: Two new steroidal saponins from Allium sativum and their inhibitory effects on blood coagulability. Yao Xue Xue Bao 31: 607-612, 1996.

58. Slowing K, Ganado P, Sanz M, Ruiz E, Beecher C and Tejerina T: Study of garlic extract and fractions on cholesterol plasma levels and vascular reactivity in cholesterol-fed rats. J Nutr 131 (Suppl 3): 994S-999S, 2001.

59. Itakura $Y$, Ichikawa M, Mori Y, Okino R, Udayama $M$ and Morita T: How to distinguish garlic from other Allium vegetables. J Nutr 131 (Suppl 3): 963S-967S, 2001.

60. Kang SS, Lim DR and Kyung KH: 3-(Allyltrisulfanyl)2-aminopropanoic acid, a novel nonvolatile water-soluble antimicrobial sulfur compound in heated garlic. J Med Food 13: 1247-1253, 2010

61. Rabinkov A, Miron T, Mirelman D, Wilchek M, Glozman S, Yavin E and Weiner L: S-Allylmercaptoglutathione: The reaction product of allicin with glutathione possesses $\mathrm{SH}$-modifying and antioxidant properties. Biochim Biophys Acta 1499: 144-153, 2000.

62. Ban JO, Yuk DY, Woo KS, Kim TM, Lee US, Jeong HS, Kim DJ, Chung YB, Hwang BY, Oh KW and Hong JT: Inhibition of cell growth and induction of apoptosis via inactivation of NF-kappaB by a sulfurcompound isolated from garlic in human colon cancer cells. J Pharmacol Sci 104: 374-383, 2007.

63. Furusawa R, Goto C, Satoh M, Nomi Y and Murata M: Formation and distribution of 2,4-dihydroxy-2,5-dimethyl-3(2H)-thiophenone, a pigment, an aroma and a biologically active compound formed by the Maillard reaction, in foods and beverages. Food Funct 4: 1076-1081, 2013.

64. Yamakawa T,Matsutomo T,Hofmann T and Kodera Y: Aged garlic extract and one of the constituent, (+)-(2S,3R)-dehydrodiconiferyl alcohol, inhibits alkaline phosphatase activity induced by inflammation factors in human vascular smooth muscle cells. Food Nutr Sci 5: 177-184, 2014 
65. Ichikawa M, Ryu K, Yoshida J, Ide N, Kodera Y, Sasaoka T and Rosen RT: Identification of six phenylpropanoids from garlic skin as major antioxidants. J Agric Food Chem 51: 7313-7317, 2003.

66. Kodera Y, Matsuura H, Yoshida S, Sumida T, Itakura Y, Fuwa T and Nishino H: Allixin, a stress compound from garlic. Chem Pharm Bull (Tokyo) 37: 1656-1658, 1989.

67. Kodera Y, Ayaba M, Ogasawara K and Ono K: Allixin induction and accumulation by light irradiation. Chem Pharm Bull (Tokyo) 49: 1636-1637, 2001.

68. Kodera Y, Ayaba M, Ogasawara K, Yoshida S, Hayashi N and Ono K: Allixin accumulation with long-term storage of garlic. Chem Pharm Bull (Tokyo) 50: 405-407, 2002.
69. Nishino H, Nishino A, Takayasu J, Iwashima A, Itakura Y, Kodera Y, Matsuura $\mathrm{H}$ and Fuwa T: Antitumor-promoting activity of allixin, a stress compound produced by garlic. Cancer J 3: 20-21, 1990.

70. Moriguchi T, Matsuura H, Itakura Y, Katsuki H, Saito H and Nishiyama N: Allixin, a phytoalexin produced by garlic, and its analogues as novel exogenous substances with neurotrophic activity. Life Sci 61: 1413-1420, 1997.

This work is licensed under a Creative Commons

Attribution-NonCommercial-NoDerivatives 4.0 International (CC BY-NC-ND 4.0) License. 